\title{
High Degree Cubature Federated Filter for Multisensor Information Fusion with Correlated Noises
}

\author{
Lijun Wang and Sisi Wang \\ School of Navigation, Guangdong Ocean University, No. 40, Jiefangdong Road, Zhanjiang 524025, China \\ Correspondence should be addressed to Sisi Wang; mars32lin@sina.com
}

Received 26 November 2015; Revised 22 April 2016; Accepted 9 August 2016

Academic Editor: Qingling Zhang

Copyright (C) 2016 L. Wang and S. Wang. This is an open access article distributed under the Creative Commons Attribution License, which permits unrestricted use, distribution, and reproduction in any medium, provided the original work is properly cited.

\begin{abstract}
This paper proposes an improved high degree cubature federated filter for the nonlinear fusion system with cross-correlation between process and measurement noises at the same time using the fifth-degree cubature rule and the decorrelated principle in its local filters. The master filter of the federated filter adopts the no-reset mode to fuse local estimates of local filters to generate a global estimate according to the scalar weighted rule. The air-traffic maneuvering target tracking simulations are performed between the proposed filter and the fifth-degree cubature federated filter. Simulations results demonstrate that the proposed filter not only can achieve almost the same accuracy as the fifth-degree cubature federated filter with independent white noises, but also has superior performance to the fifth-degree cubature federated filter while the noises are cross-correlated at the same time.
\end{abstract}

\section{Introduction}

Federated filter (FF) proposed by Carlson has been further studied and widely applied in many multisensor information fusion systems [1-3], that is, target tracking system and integrated navigation system. FF can achieve improved accuracy and more specific inferences in fusing the data provided by different sensors, such as radar, automatic identification system, global positioning system, celestial navigation system, inertial navigation system, dead reckoning system, and infrared [1-6].

For traditional FF, it is assumed that the process noise and measurement noise in fusion systems are of statistical independence. However, the above noise assumptions are often violated in practice; for example, in target tracking fusion systems, discretization of real continuous fusion systems may bring about cross-correlation between the process and measurement noises. For target tracking fusion systems, there would exist some cross-correlation between the process and measurement noises if both of them are dependent on the system states [7]. It is evident that the global approximated optimality of traditional FF is difficult to obtain when the system noises are cross-correlated. Consequently, it is necessary to develop improved FF with cross-correlated noises.

FF is initially developed to solve optimal estimation problems with linear Kalman filter (KF) as its local filter. However, in nonlinear fusion cases, global performance of the filter mainly depends on the selection of its nonlinear local filter, and the linear KF cannot work well when dynamic and measurement models in fusion systems are actually nonlinear. Until now, research works on information fusion with crosscorrelated noises are mainly concentrated on the corresponding linear fusion filter. For example, an optimal KF is presented for linear dynamic systems with sensor noises crosscorrelated [8] and a distributed weighted robust KF fusion is studied for a class of uncertain systems with autocorrelated and cross-correlated noises [7]. The proposed FF aims to fuse information from nonlinear models with cross-correlated noises, so a suitable nonlinear filter should be used as the local filter of FF instead of the KF. Compared with other classical nonlinear filters, the high degree cubature Kalman filter (HCKF) seems to be a good choice to be the substituted local filter, which has superior performance to the extend Kalman filter (EKF), the cubature Kalman filter (CKF), the unscented Kalman filter (UKF), and the particle filter (PF) 
[9-12]. To the best knowledge of the authors' knowledge, no works about the high degree cubature federated filters with correlated noises (HCFF-CNs) have been reported.

The solution is proposed by constructing the framework of HCFF-CNs on the basis of nonlinear FF with correlated noises at the same time and the fifth-degree sphericalradial rule. The global filter of HCFF-CNs still adopts the fusion-reset mode to keep good fusion accuracy. In the end, simulation contrast tests are done to verify the effectiveness of the filters. The rest of the paper is organized as follows. The basic knowledge is formulated in Section 2. In Section 3, the HCFF-CNs is proposed. Contrast tests of maneuvering target tracking fusion are provided in Section 4. Section 5 concludes the paper.

\section{Preliminaries}

2.1. System Description. Considering a kind of nonlinear discrete dynamic stochastic system,

$$
\begin{aligned}
& \mathbf{x}_{k+1}=f_{k}\left(\mathbf{x}_{k}\right)+\mathbf{w}_{k}, \\
& \mathbf{z}_{m, k}=h_{m, k}\left(\mathbf{x}_{k}\right)+\mathbf{v}_{m, k},
\end{aligned}
$$

where $k \in \mathbb{N}$ is a discrete sample time index; $m=1,2, \ldots, N$ is the sensor index. $\mathbf{x}_{k} \in \mathbb{R}^{n}$ and $\mathbf{z}_{m, k} \in \mathbb{R}^{p_{m}}$ are the system state and the $m$ th measurement vectors at time index $k$, respectively; $f_{k}(\cdot)$ and $h_{m, k}(\cdot)$ are known nonlinear dynamic and measurement equations at time index $k$, respectively; process noise sequence $\left\{\mathbf{w}_{k}\right\}$ and measurement noise sequence $\left\{\mathbf{v}_{m, k}\right\}$ are correlated zero-mean Gaussian white noise sequences satisfying

$$
\begin{aligned}
\mathrm{E}\left[\mathbf{w}_{k} \mathbf{w}_{j}^{\mathrm{T}}\right] & =\mathbf{Q}_{k} \delta_{k j}, \\
\mathrm{E}\left[\mathbf{v}_{m, k} \mathbf{v}_{m, l}^{\mathrm{T}}\right] & =\mathbf{R}_{m, k} \delta_{k l}, \\
\mathrm{E}\left[\mathbf{w}_{k} \mathbf{v}_{m, l}^{\mathrm{T}}\right] & =\mathbf{D}_{m, k} \delta_{k l},
\end{aligned}
$$

where $\delta_{k l}$ is Kronecker delta function. The initial state is $\mathbf{x}_{0}$ and its associated mean and covariance are $\widehat{\mathbf{x}}_{0 \mid 0}$ and $\mathbf{P}_{0 \mid 0} \cdot \mathbf{x}_{0}$ is uncorrelated with $\left\{\mathbf{w}_{k}\right\}$ and $\left\{\mathbf{v}_{m, k}\right\}$. Note that all the noise cross-correlation in the rest of this paper means the process noise and measurement noise are cross-correlated at the same time.

2.2. Problem Statement. It is evident that the traditional HCFF is inapplicable to nonlinear system described in (1) which has cross-correlative process and measurement noises. The goal of this paper is to design HCFF-CNs to suit the system with higher accuracy and stability. The main contributions of this note include the following.

(i) Nonlinear FF with cross-correlated noises (NFF$\mathrm{CNs}$ ) is constructed.

(ii) HCFF-CNs based on NFF-CNs and fifth-degree cubature rule is proposed.

(iii) HCFF-CNs achieves close accuracy to HCFF when noises are cross-independent. (iv) HCFF-CNs achieves higher accuracy than HCFF when noises are cross-correlative.

\section{Design of HCFF-CNs Algorithm}

In this section, a framework of NFF-CNs is constructed, and then HCFF-CNs is proposed.

3.1. Framework of Nonlinear FF with Cross-Correlated Noises. Like traditional FF, NFF-CNs still is a two-stage data fusion technique. Sensor-related filters are subsequently processed and combined by a larger master filter. The NFF-CNs architecture is illustrated in Figure 1, where it is operating in its fusion-reset (FR) mode.

Figure 1 shows the major flow of information but does not attempt to represent all the possible data exchanges among components. As suggested by Figure 1, each local filter uses data from multisources including common reference system, each local sensor, and the master filter. Considering the nonlinearity and the noises cross-correlation of subsystems, all the local filters use nonlinear filters with correlated noises (NFCN), where the global state $\widehat{\mathbf{x}}^{g}$ and its associated covariance $\mathbf{P}^{g}$ is fed back. Therefore, the selection of local filters directly affects the global performance of HCFF-CNs. The structure and function of master filter are similar to traditional FF.

3.2. The Fifth-Degree Cubature Rule. Similar to the thirddegree cubature rule, the fifth-degree cubature rule is still approximated efficiently to the Gaussian weighted integrals by a set of corresponding fifth-degree cubature points and weights. The dimension of nonlinear discrete system is defined as $n$, the points to calculate the radial rule are not equal to zero, and the number of radial rule point is 2 ; then the number of spherical rule point is defined as $2 n^{2}$, and the total number of fifth-degree cubature points is $2 n^{2}+1$. The corresponding cubature points and weights are given as follows:

$$
\begin{aligned}
\boldsymbol{\varsigma}_{i} & =[0, \ldots, 0]^{\mathrm{T}}, \\
W_{i} & =\frac{2}{(n+2)}, \quad i=0, \\
\boldsymbol{\varsigma}_{i}^{+} & =\sqrt{n+2} \mathbf{s}_{i}^{+}, \\
\boldsymbol{\varsigma}_{i}^{-} & =\sqrt{n+2} \mathbf{s}_{i}^{-}, \\
W_{i} & =\frac{1}{(n+2)^{2}}, \\
\boldsymbol{\varsigma}_{i}^{+} & =-\sqrt{n+2} \mathbf{s}_{i-n(n-1) / 2}^{+}, \\
\boldsymbol{\varsigma}_{i}^{-} & =-\sqrt{n+2} \mathbf{s}_{i-n(n-1) / 2}^{-},
\end{aligned}
$$




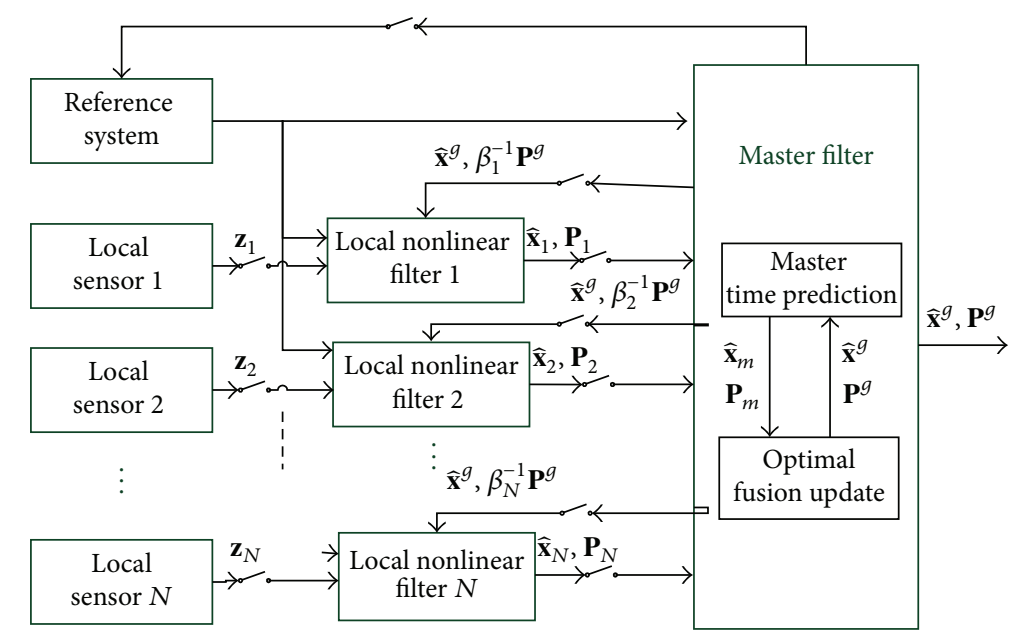

FIGURE 1: Nonlinear federated filter with cross-correlated noise (fusion-reset mode).

$W_{i}=\frac{1}{(n+2)^{2}}$,

$$
\frac{n(n-1)}{2}+1 \leq i \leq n(n-1)
$$

$\boldsymbol{\varsigma}_{j}^{e}=\sqrt{n+2} \mathbf{e}_{j}$,

$W_{j}=\frac{(4-n)}{\left[2(n+2)^{2}\right]}$,

$$
1 \leq j \leq n,
$$

$\boldsymbol{\varsigma}_{j}^{e}=-\sqrt{n+2} \mathbf{e}_{j-n}$

$W_{j}=\frac{(4-n)}{\left[2(n+2)^{2}\right]}$, theoretically the equivalence of the above two classes of method in linear and nonlinear systems $[16,17]$. Due to the equivalence between the two classes of filters, the GASFCNs is chosen as the local nonlinear filter for decorrelation between the process and each measurement noise. And then the fifth-degree cubature rule is used to approximate the local filter which is constructed by GASF-CNs. The whole framework of local filter is summarized as follows.

(1) The one-step predicted mean and covariance of initial state $\mathbf{x}_{m, 0}$ are both known, which is roughly equal to $\widehat{\mathbf{x}}_{m, 0 \mid 0}$ and $\mathbf{P}_{m, 0 \mid 0}$.

(2) Evaluate predicted states $\widehat{\mathbf{x}}_{m, k+1 \mid k}$ and $\mathbf{P}_{m, k+1 \mid k}$

$$
\begin{aligned}
\widehat{\mathbf{x}}_{m, k+1 \mid k} & =\widehat{\mathbf{x}}_{m, k+1 \mid k-1}+\mathbf{W}_{m, k}\left(\mathbf{z}_{m, k}-\widehat{\mathbf{z}}_{m, k \mid k-1}\right), \\
\mathbf{P}_{m, k+1 \mid k} & =\mathbf{P}_{m, k+1 \mid k-1}-\mathbf{W}_{m, k} \mathbf{P}_{m, k \mid k-1}^{\mathbf{z z}} \mathbf{W}_{m, k}^{\mathrm{T}}, \\
\mathbf{W}_{m, k} & =\mathbf{P}_{m, k+1, k \mid k-1}^{\mathbf{x z}}\left(\mathbf{P}_{m, k \mid k-1}^{\mathbf{z z}}\right)^{-1},
\end{aligned}
$$

where $\mathbf{e}_{j}$ is the unit vector in $\mathbb{R}^{n}$ with the $i$ th element being one. The points of $\mathbf{s}_{i}^{+}$and $\mathbf{s}_{i}^{-}$are given by

$$
\begin{aligned}
& \left\{\mathbf{s}_{i}^{+}\right\} \triangleq\left\{\sqrt{\frac{1}{2}}\left(\mathbf{e}_{k}+\mathbf{e}_{l}\right): k<l, k, l=1,2, \ldots, n\right\}, \\
& \left\{\mathbf{s}_{i}^{-}\right\} \triangleq\left\{\sqrt{\frac{1}{2}}\left(\mathbf{e}_{k}-\mathbf{e}_{l}\right): k<l, k, l=1,2, \ldots, n\right\} .
\end{aligned}
$$

3.3. Local Filter of HCFF-CNs. In the past decades, the nonlinear filters for cross-correlated noises are mainly divided into two classes. One can decorrelate the noise sequences directly using the conventional method introduced from linear KF with cross-correlated noises [13], and the other is Gaussian approximation recursive filter with correlated noises (GASF-CNs), which is extended to the UKF with correlative noises $[14,15]$. Furthermore, Chang et al. derived where the two-step state prediction $\widehat{\mathbf{x}}_{k+1 \mid k-1}$ and associated covariance $\mathbf{P}_{k+1 \mid k-1}$ are given by

$$
\begin{aligned}
\widehat{\mathbf{x}}_{m, k+1 \mid k-1}=\int_{\mathbb{R}^{n}} f_{k}\left(\mathbf{x}_{m, k}\right) \\
\cdot N\left(\mathbf{x}_{m, k} ; \widehat{\mathbf{x}}_{m, k \mid k-1}, \mathbf{P}_{m, k \mid k-1}\right) \mathrm{d} \mathbf{x}_{m, k} \\
=\frac{2}{n+2} f_{k}\left(\widehat{\mathbf{x}}_{m, k \mid k-1}\right)+\frac{1}{(n+2)^{2}} \\
\cdot \sum_{i=1}^{n(n-1)}\left(f_{k}\left(\mathbf{X}_{m, i, k \mid k-1}^{+}\right)+f_{k}\left(\mathbf{X}_{m, i, k \mid k-1}^{-}\right)\right) \\
+\frac{4-n}{2(n+2)^{2}} \sum_{j=1}^{2 n} f_{k}\left(\mathbf{X}_{m, j, k \mid k-1}\right),
\end{aligned}
$$




$$
\begin{aligned}
\mathbf{P}_{m, k+1 \mid k-1}=\int_{\mathbb{R}^{n}} f_{k}\left(\mathbf{x}_{m, k}\right) f_{k}^{\mathrm{T}}\left(\mathbf{x}_{m, k}\right) \\
\cdot N\left(\mathbf{x}_{m, k} ; \widehat{\mathbf{x}}_{m, k \mid k-1}, \mathbf{P}_{m, k \mid k-1}\right) \mathrm{d} \mathbf{x}_{m, k} \\
-\widehat{\mathbf{x}}_{m, k+1 \mid k-1} \widehat{\mathbf{x}}_{m, k+1 \mid k-1}^{\mathrm{T}}+\mathbf{Q}_{m, k}=\frac{2}{n+2} f_{k}\left(\widehat{\mathbf{x}}_{m, k \mid k-1}\right) \\
\cdot\left(f_{k}\left(\widehat{\mathbf{x}}_{m, k \mid k-1}\right)\right)^{\mathrm{T}}+\frac{1}{(n+2)^{2}} \\
\quad \cdot \sum_{i=1}^{n(n-1)}\left(f_{k}\left(\mathbf{X}_{m, i, k \mid k-1}^{+}\right)\left(f_{k}\left(\mathbf{X}_{m, i, k \mid k-1}^{+}\right)\right)^{\mathrm{T}}\right. \\
\left.+f_{k}\left(\mathbf{X}_{m, i, k \mid k-1}^{-}\right)\left(f_{k}\left(\mathbf{X}_{m, i, k \mid k-1}^{-}\right)\right)^{\mathrm{T}}\right)+\frac{4-n}{2(n+2)^{2}} \\
\quad \cdot \sum_{j=1}^{2 n} f_{k}\left(\mathbf{X}_{m, j, k \mid k-1}\right)\left(f_{k}\left(\mathbf{X}_{m, j, k \mid k-1}\right)\right)^{\mathrm{T}} \\
-\widehat{\mathbf{x}}_{m, k+1 \mid k-1} \widehat{\mathbf{x}}_{m, k+1 \mid k-1}^{\mathrm{T}}+\mathbf{Q}_{m, k},
\end{aligned}
$$

where $N(\cdot ; \cdot, \cdot)$ is the conventional symbol for Gaussian density. The one-step predicted measurement and innovation covariance of $\mathbf{z}_{m, k}$ and the cross-covariance $\mathbf{P}_{m, \mathbf{x z}, k+1, k \mid k-1}$ are given by

$$
\begin{aligned}
& \widehat{\mathbf{z}}_{m, k \mid k-1}=\int_{\mathbb{R}^{n}} h_{m, k}\left(\mathbf{x}_{m, k}\right) \\
& \cdot N\left(\mathbf{x}_{m, k} ; \widehat{\mathbf{x}}_{m, k \mid k-1}, \mathbf{P}_{m, k \mid k-1}\right) \mathrm{d} \mathbf{x}_{m, k} \\
& =\frac{2}{n+2} h_{m, k}\left(\widehat{\mathbf{x}}_{m, k \mid k-1}\right)+\frac{1}{(n+2)^{2}} \\
& \cdot \sum_{i=1}^{n(n-1)}\left(h_{m, k}\left(\mathbf{X}_{m, i, k \mid k-1}^{+}\right)+h_{m, k}\left(\mathbf{X}_{m, i, k \mid k-1}^{-}\right)\right) \\
& +\frac{4-n}{2(n+2)^{2}} \sum_{j=1}^{2 n} h_{m, k}\left(\mathbf{X}_{m, j, k \mid k-1}\right) \\
& \mathbf{P}_{m, k \mid k-1}^{\mathbf{z z}}=\int_{\mathbb{R}^{n}} h_{m, k}\left(\mathbf{x}_{m, k}\right) h_{k}^{\mathrm{T}}\left(\mathbf{x}_{m, k}\right) \\
& \cdot N\left(\mathbf{x}_{m, k} ; \widehat{\mathbf{x}}_{m, k \mid k-1}, \mathbf{P}_{m, k \mid k-1}\right) \mathrm{d} \mathbf{x}_{m, k} \\
& -\widehat{\mathbf{z}}_{m, k \mid k-1} \widehat{\mathbf{z}}_{m, k \mid k-1}^{\mathrm{T}}+\mathbf{R}_{m, k}=\frac{2}{n+2} h_{m, k}\left(\widehat{\mathbf{x}}_{m, k \mid k-1}\right) \\
& \cdot\left(h_{m, k}\left(\widehat{\mathbf{x}}_{m, k \mid k-1}\right)\right)^{\mathrm{T}}+\frac{1}{(n+2)^{2}} \\
& \cdot \sum_{i=1}^{n(n-1)}\left(h_{m, k}\left(\mathbf{X}_{m, i, k \mid k-1}^{+}\right)\left(h_{m, k}\left(\mathbf{X}_{m, i, k \mid k-1}^{+}\right)\right)^{\mathrm{T}}\right. \\
& \left.+h_{m, k}\left(\mathbf{X}_{m, i, k \mid k-1}^{-}\right)\left(h_{m, k}\left(\mathbf{X}_{m, i, k \mid k-1}^{-}\right)\right)^{\mathrm{T}}\right) \\
& +\frac{4-n}{2(n+2)^{2}} \sum_{j=1}^{2 n} h_{m, k}\left(\mathbf{X}_{m, j, k \mid k-1}\right)\left(h_{m, k}\left(\mathbf{X}_{m, j, k \mid k-1}\right)\right)^{\mathrm{T}} \\
& -\widehat{\mathbf{z}}_{m, k \mid k-1} \widehat{\mathbf{z}}_{m, k \mid k-1}^{\mathrm{T}}+\mathbf{R}_{m, k},
\end{aligned}
$$

$$
\begin{aligned}
& \mathbf{P}_{m, k+1, k \mid k-1}^{\mathbf{x z}}=\int_{\mathbb{R}^{n}} f_{k}\left(\mathbf{x}_{m, k}\right) h_{k}^{\mathrm{T}}\left(\mathbf{x}_{m, k}\right) \\
& \cdot N\left(\mathbf{x}_{m, k} ; \widehat{\mathbf{x}}_{m, k \mid k-1}, \mathbf{P}_{m, k \mid k-1}\right) \mathrm{d} \mathbf{x}_{k} \\
& -\widehat{\mathbf{x}}_{m, k+1 \mid k-1} \widehat{\mathbf{z}}_{m, k \mid k-1}^{\mathrm{T}}+\mathbf{D}_{m, k}=\frac{2}{n+2} f_{k}\left(\widehat{\mathbf{x}}_{m, k \mid k-1}\right) \\
& \cdot\left(h_{m, k}\left(\widehat{\mathbf{x}}_{m, k \mid k-1}\right)\right)^{\mathrm{T}}+\frac{1}{(n+2)^{2}} \\
& \quad \cdot \sum_{i=1}^{n(n-1)}\left(f_{k}\left(\mathbf{X}_{m, i, k \mid k-1}^{+}\right)\left(h\left(\mathbf{X}_{m, i, k \mid k-1}^{+}\right)\right)^{\mathrm{T}}\right. \\
& \left.+f_{k}\left(\mathbf{X}_{m, i, k \mid k-1}^{-}\right)\left(h_{k}\left(\mathbf{X}_{m, i, k \mid k-1}^{-}\right)\right)^{\mathrm{T}}\right)+\frac{4-n}{2(n+2)^{2}} \\
& \quad \cdot \sum_{j=1}^{2 n} f_{k}\left(\mathbf{X}_{m, j, k \mid k-1}\right)\left(h_{k}\left(\mathbf{X}_{m, j, k \mid k-1}\right)\right)^{\mathrm{T}}
\end{aligned}
$$$$
-\widehat{\mathbf{x}}_{m, k+1 \mid k-1} \widehat{\mathbf{z}}_{m, k \mid k-1}^{\mathrm{T}}+\mathbf{D}_{m, k}
$$

where the cubature points in (7) are defined as follows:

$$
\begin{aligned}
& \mathbf{X}_{m, i, k \mid k-1}^{+}=\mathbf{S}_{m, k \mid k-1} \boldsymbol{\varsigma}_{i}^{+}+\widehat{\mathbf{x}}_{m, k \mid k-1}, \\
& \mathbf{X}_{m, i, k \mid k-1}^{-}=\mathbf{S}_{m, k \mid k-1} \boldsymbol{\zeta}_{i}^{-}+\widehat{\mathbf{x}}_{m, k \mid k-1}, \\
& \mathbf{X}_{m, j, k \mid k-1}=\mathbf{S}_{m, k \mid k-1} \boldsymbol{\varsigma}_{j}^{e}+\widehat{\mathbf{x}}_{m, k \mid k-1}
\end{aligned}
$$

where $\mathbf{P}_{m, k \mid k-1}=\mathbf{S}_{m, k \mid k-1} \mathbf{S}_{m, k \mid k-1}^{\mathrm{T}}$ and $\mathbf{S}_{m, k \mid k-1}$ can be obtained by the Cholesky decomposition or the singular value decomposition.

(3) Update the mean and covariance of $\mathbf{x}_{k+1}$.

$$
\begin{aligned}
& \widehat{\mathbf{x}}_{m, k+1 \mid k+1}=\widehat{\mathbf{x}}_{m, k+1 \mid k}+\mathbf{K}_{m, k+1}\left(\mathbf{z}_{m, k+1}-\widehat{\mathbf{z}}_{m, k+1 \mid k}\right), \\
& \mathbf{P}_{m, k+1 \mid k+1}=\mathbf{P}_{m, k+1 \mid k}-\mathbf{K}_{m, k+1} \mathbf{P}_{m, \mathbf{z z}, k+1 \mid k} \mathbf{K}_{m, k+1}^{\mathrm{T}},
\end{aligned}
$$

where filtering gain $\mathbf{K}_{m, k+1}=\mathbf{P}_{m, \mathbf{x z}, k+1 \mid k} \mathbf{P}_{m, \mathbf{z z}, k+1 \mid k}^{-1}$. The predicted measurement $\widehat{\mathbf{z}}_{m, k+1 \mid k}$, the innovation covariance $\mathbf{P}_{m, \mathbf{z z}, k+1 \mid k}$, and the cross-covariance $\mathbf{P}_{m, \mathbf{x z}, k+1 \mid k}$ are given by

$$
\begin{aligned}
& \widehat{\mathbf{z}}_{m, k+1 \mid k}=\frac{2}{n+2} h\left(\widehat{\mathbf{x}}_{m, k+1 \mid k}\right)+\frac{4-n}{2(n+2)^{2}} \\
& \cdot \sum_{j=1}^{2 n} h\left(\mathbf{X}_{m, j, k+1 \mid k}\right)+\frac{1}{(n+2)^{2}} \sum_{i=1}^{n(n-1)}\left(h\left(\mathbf{X}_{m, i, k+1 \mid k}^{+}\right)\right. \\
& \left.+h\left(\mathbf{X}_{m, i, k+1 \mid k}^{-}\right)\right),
\end{aligned}
$$




$$
\begin{aligned}
& \mathbf{P}_{m, \mathbf{z z}, k+1 \mid k}=\frac{2}{n+2} h\left(\widehat{\mathbf{x}}_{m, k+1 \mid k}\right)\left(h\left(\widehat{\mathbf{x}}_{m, k+1 \mid k}\right)\right)^{\mathrm{T}} \\
& +\frac{1}{(n+2)^{2}} \sum_{i=1}^{n(n-1)}\left(h\left(\mathbf{X}_{m, i, k+1 \mid k}^{+}\right)\left(h\left(\mathbf{X}_{m, i, k+1 \mid k}^{+}\right)\right)^{\mathrm{T}}\right. \\
& \left.+h\left(\mathbf{X}_{m, i, k+1 \mid k}^{-}\right)\left(h\left(\mathbf{X}_{m, i, k+1 \mid k}^{-}\right)\right)^{\mathrm{T}}\right)+\frac{4-n}{2(n+2)^{2}} \\
& \quad \cdot \sum_{j=1}^{2 n} h\left(\mathbf{X}_{m, j, k+1 \mid k}\right)\left(h\left(\mathbf{X}_{m, j, k+1 \mid k}\right)\right)^{\mathrm{T}} \\
& \quad-\widehat{\mathbf{z}}_{m, k+1 \mid k}\left(\widehat{\mathbf{z}}_{m, k+1 \mid k}\right)^{\mathrm{T}}+\mathbf{R}_{m, k+1}, \\
& \mathbf{P}_{\mathbf{x z}, k+1 \mid k}=\frac{2}{n+2} \widehat{\mathbf{x}}_{m, k+1 \mid k}\left(h\left(\widehat{\mathbf{x}}_{m, k+1 \mid k}\right)\right)^{\mathrm{T}}+\frac{1}{(n+2)^{2}} \\
& \quad+\sum_{i=1}^{n(n-1)}\left(\mathbf{X}_{m, i, k+1 \mid k}^{+}\left(h\left(\mathbf{X}_{m, i, k+1 \mid k}^{+}\right)\right)^{\mathrm{T}}\right. \\
& \left.\quad+\mathbf{X}_{m, i, k+1 \mid k}^{-}\left(h\left(\mathbf{X}_{m, i, k+1 \mid k}^{-}\right)\right)^{\mathrm{T}}\right)+\frac{4-n}{2(n+2)^{2}} \\
& \quad \sum_{j=1}^{2 n} \mathbf{X}_{j, k+1 \mid k}\left(h\left(\mathbf{X}_{m, j, k+1 \mid k}\right)\right)^{\mathrm{T}}-\widehat{\mathbf{x}}_{m, k+1 \mid k}\left(\widehat{\mathbf{z}}_{m, k+1 \mid k}\right)^{\mathrm{T}},
\end{aligned}
$$

where the propagated cubature points in (10) are defined as

$$
\begin{aligned}
& \mathbf{X}_{m, i, k+1 \mid k}^{+}=\mathbf{S}_{m, k+1 \mid k} \boldsymbol{\zeta}_{i}^{+}+\widehat{\mathbf{x}}_{m, k+1 \mid k}, \\
& \mathbf{X}_{m, i, k+1 \mid k}^{-}=\mathbf{S}_{m, k+1 \mid k} \boldsymbol{\zeta}_{i}^{-}+\widehat{\mathbf{x}}_{m, k+1 \mid k}, \\
& \mathbf{X}_{m, j, k+1 \mid k}=\mathbf{S}_{m, k+1 \mid k} \boldsymbol{\zeta}_{j}^{e}+\widehat{\mathbf{x}}_{m, k+1 \mid k}
\end{aligned}
$$

where $\mathbf{P}_{m, k+1 \mid k}=\mathbf{S}_{m, k+1 \mid k} \mathbf{S}_{m, k+1 \mid k}^{\mathrm{T}}$ and $\mathbf{S}_{m, k+1 \mid k}$ can be obtained similar to $\mathbf{S}_{m, k \mid k-1}$.

3.4. Global Fusion Algorithm. In order to efficiently recombine and fuse the local filter outputs and obtain an enhanced estimation result, the scalar weighted optimal fusion method is used. The FF can be presented with the following steps.

\section{(1) Information Distribution.}

$$
\begin{aligned}
\mathbf{Q}_{i, k} & =\beta_{i}^{-1} \mathbf{Q}_{k}, \\
\mathbf{P}_{i, k \mid k} & =\beta_{i}^{-1} \mathbf{P}_{k \mid k}^{g}, \\
\widehat{\mathbf{x}}_{i, k \mid k} & =\widehat{\mathbf{x}}_{k \mid k}^{g},
\end{aligned}
$$

$$
i=1,2, \ldots, N, M \text {, }
$$

where $\beta_{i}=1 / N$ and $\beta_{M}=0$ are the information distribution coefficients of each local filter and master filter, which satisfy $\sum_{i=1}^{N} \beta_{i}+\beta_{M}=0$.
(2) Information Update. Prediction and update steps which are performed in each local filter are described in Section 3.3.

(3) Information Fusion.

$$
\begin{aligned}
& \mathbf{P}_{k+1 \mid k+1}^{g}=\left[\sum_{m=1}^{N} \mathbf{P}_{m, k+1 \mid k+1}^{-1}\right]^{-1}, \\
& \widehat{\mathbf{x}}_{k+1 \mid k+1}^{g}=\mathbf{P}_{k+1 \mid k+1}^{g}\left[\sum_{m=1}^{N} \mathbf{P}_{m, k+1 \mid k+1}^{-1} \widehat{\mathbf{x}}_{m, k+1 \mid k+1}\right]^{-1} .
\end{aligned}
$$

\section{Simulation Examples}

4.1. Target Tracking. A classic maneuvering target tracking application is considered, which executes maneuvering turn in a horizontal plane at a known and constant turn rate [2]. The kinematic of the turning motion and measurement model can be generalized by the following equations:

$$
\begin{aligned}
\mathbf{x}_{k+1}= & {\left[\begin{array}{cccc}
1 & \frac{\sin \Omega \Delta t}{\Omega} & 0 & -\left(\frac{1-\cos \Omega \Delta t}{\Omega}\right) \\
0 & \cos \Omega \Delta t & 0 & -\sin \Omega \Delta t \\
0 & \frac{1-\cos \Omega \Delta t}{\Omega} & 1 & \frac{\sin \Omega \Delta t}{\Omega} \\
0 & \sin \Omega \Delta t & 0 & \cos \Omega \Delta t
\end{array}\right] \mathbf{x}_{k} } \\
& +\mathbf{w}_{k}, \quad\left[\begin{array}{l}
\sqrt{\left(x_{k}-x_{s}\right)^{2}+\left(y_{k}-y_{s}\right)^{2}} \\
\mathbf{z}_{m, k}=
\end{array}\right]+\mathbf{v}_{m, k},
\end{aligned}
$$

where the target state $\mathbf{x}_{k}=\left[\begin{array}{llll}x_{k} & \dot{x}_{k} & y_{k} & \dot{y}_{k}\end{array}\right]^{\mathrm{T}} ; x_{k}$ and $y_{k}$ denote the positions and $\dot{x}_{k}$ and $\dot{y}_{k}$ denote the velocities in $x$ and $y$ directions, respectively; $x_{s}$ and $y_{s}$ denote the positions of radar installing and three radars are used as tracking sensors; $\Omega$ is a known and constant turn rate; $\Delta t$ is the time interval between two consecutive measurements; the process noise $\mathbf{w}_{k}$ and measurement noise $\mathbf{v}_{m, k}$ are crosscorrelated zero-mean Gaussian white noise with covariance $\mathbf{Q}_{k}$ and $\mathbf{R}_{m, k}$, and $\mathbf{Q}_{k}$ satisfies

$$
\mathbf{Q}_{k}=\mathrm{E}\left[\mathbf{w}_{k} \mathbf{w}_{k}^{\mathrm{T}}\right]=\left[\begin{array}{cccc}
\frac{\Delta t^{3}}{3} & \frac{\Delta t^{2}}{2} & 0 & 0 \\
\frac{\Delta t^{2}}{2} & \Delta t & 0 & 0 \\
0 & 0 & \frac{\Delta t^{3}}{3} & \frac{\Delta t^{2}}{2} \\
0 & 0 & \frac{\Delta t^{2}}{2} & \Delta t
\end{array}\right]
$$




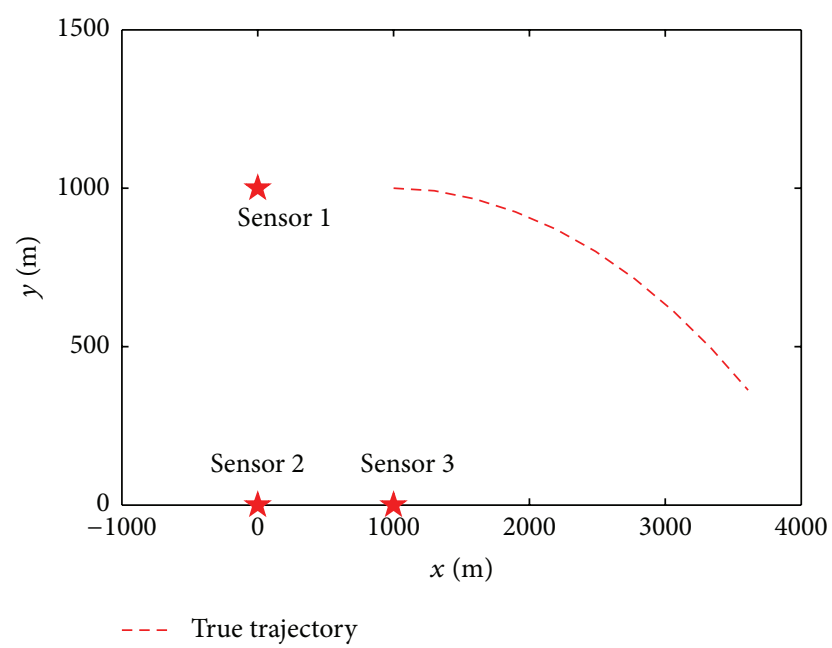

FIGURE 2: Sensors positions and target true trajectory.

True initial state, its associate covariance, and other parameters are defined as follows:

$$
\begin{aligned}
\mathbf{x}_{0} & =\left[\begin{array}{llll}
1000 \mathrm{~m} & 300 \mathrm{~ms}^{-1} & 1000 \mathrm{~m} & 0 \mathrm{~ms}^{-1}
\end{array}\right]^{\mathrm{T}}, \\
\mathbf{P}_{0 \mid 0} & =\operatorname{diag}\left[\begin{array}{llll}
100 \mathrm{~m}^{2} & 10 \mathrm{~m}^{2} \mathrm{~s}^{-2} & 100 \mathrm{~m}^{2} & 10 \mathrm{~m}^{2} \mathrm{~s}^{-2}
\end{array}\right], \\
\Delta t & =1 \mathrm{~s}, \\
\Omega & =-3^{\circ} \mathrm{s}^{-1},
\end{aligned}
$$

where $\widehat{\mathbf{x}}_{0 \mid 0} \sim N\left(\mathbf{x}_{0}, \mathbf{P}_{0 \mid 0}\right)$. Figure 2 shows the fixing radars positions and the true trajectory during 10 sample times.

4.2. Result Analysis. For a fair comparison, independent Monte Carlo runs $(P)$ equal 150. The total number of scans per run is 10. All the filters are initialized with the same condition in each run. To compare the various nonlinear filter performances, the metric is the root mean square error (RMSE). For example, the RMSE in position at time $k+1$ is defined as

$$
\begin{aligned}
& \operatorname{RMSE}_{k+1}^{\mathrm{pos}} \\
& \quad=\sqrt{\frac{1}{P} \sum_{n=1}^{P}\left(\left(x_{k+1}-\hat{x}_{k+1}^{n}\right)^{2}+\left(y_{k+1}-\hat{y}_{k+1}^{n}\right)^{2}\right),}
\end{aligned}
$$

where $\left(x_{k+1}, y_{k+1}\right)$ is the true position at discrete time $k+1$ and $\left(\widehat{x}_{k+1}^{n}, \widehat{y}_{k+1}^{n}\right)$ is estimated position at discrete time $k+1$ of the $n$th Monte Carlo run. Similarly to the RMSE in position, RMSE in velocity can be obtained.

Scenario 1. In Scenario 1, let $\mathbf{D}_{m, k}=0$ and the covariance of $\mathbf{v}_{m, k}$ satisfies $\mathbf{R}_{m, k}=\operatorname{diag}\left[1600 \mathrm{~m}^{2} 200 \mathrm{mrad}^{2}\right] . \mathbf{D}_{m, k}=0$ implies that $\mathbf{w}_{k}$ and $\mathbf{v}_{m, k}$ are uncorrelated. The RMSE results in position and velocity of HCFF-CNs and HCFF are shown in Figure 3 and Table 1.

From Figure 3, it can be seen that the RMSE lines in position and velocity of HCFF and HCFF-CNs are very close.
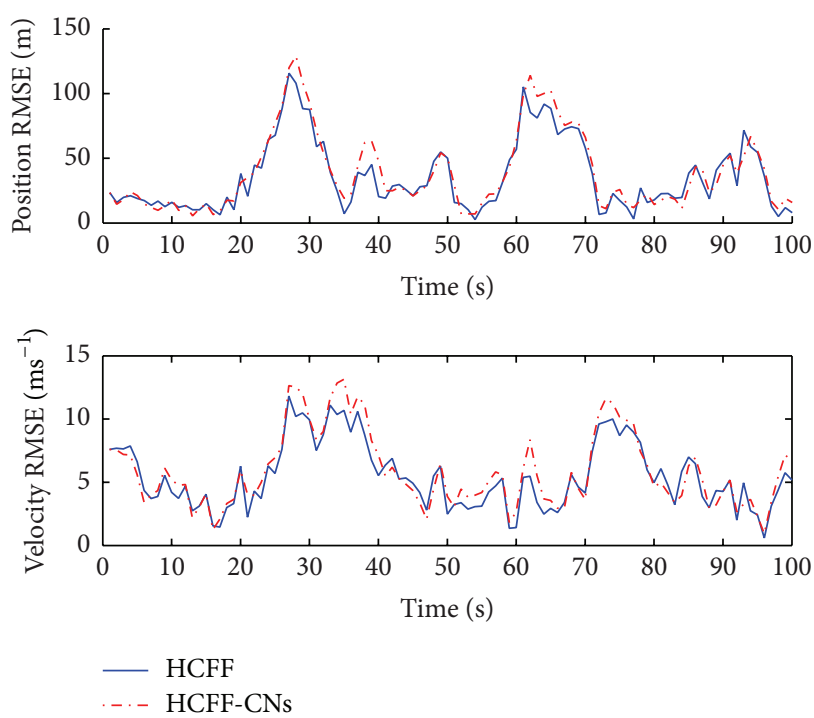

FIGURE 3: RMSEs in position and velocity for Scenario 1.

TABLE 1: Means of RMSEs in position and velocity for Scenario 1.

\begin{tabular}{lcc}
\hline Algorithms & HCFF & HCFF-CNs \\
\hline RMSE means in position $(\mathrm{m})$ & 35.737 & 36.1052 \\
RMSE means in velocity $\left(\mathrm{ms}^{-1}\right)$ & 5.4546 & 5.8804 \\
\hline
\end{tabular}

That is to say, the HCFF-CNs can operate nearly as well as the HCFF when $\mathbf{D}_{m, k}=0$. The data in Table 1 also support our analysis. In addition, considering the effect of crosscorrelated noises, the prior knowledge used in HCFF-CNs to predict $\widehat{\mathbf{x}}_{m, k+1 \mid k}$ and $\mathbf{P}_{m, k+1 \mid k}$ includes no information of $\mathbf{z}_{m, k}$, which may lead to a little accuracy degeneracy of HCFF-CNs in Scenario 1.

Scenario 2. In Scenario 2, let $\mathbf{v}_{m, k}=\mathbf{b} \mathbf{w}_{k}$, then $\mathbf{R}_{m, k}=\mathbf{b} \mathbf{Q}_{k} \mathbf{b}^{\mathrm{T}}$, and $\mathbf{D}_{m, k}=\mathbf{Q}_{k} \mathbf{b}^{\mathrm{T}} \neq \mathbf{0}[10]$. The correlation coefficient $\mathbf{b}=$

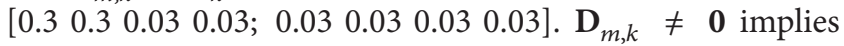
that $\mathbf{w}_{k}$ and $\mathbf{v}_{m, k}$ are cross-correlated. The RMSE results in position and velocity of HCFF-CNs and HCFF are shown in Figure 4 and Table 2.

When $\mathbf{D}_{m, k} \neq \mathbf{0}$, Figure 4 illustrates that the RMSE line in position and velocity of traditional HCFF far exceed the corresponding lines of the proposed filter. It implies that in Scenario $2 \mathrm{HCFF}-\mathrm{CNs}$ can improve the performance of $\mathrm{HCFF}$ whose accuracy and robustness are badly affected by the cross-correlation of process noise and measurement noise. The data in Table 2 supports the same deduction.

\section{Conclusions}

Aiming at traditional FF which is not applicable to the nonlinear discrete dynamic stochastic system with crosscorrelative noises, an improved FF is proposed. The simulation results demonstrate that the proposed filter has nearly the same performance as the HCFF when the process noise and measurement noise are independent. Moreover, it can 


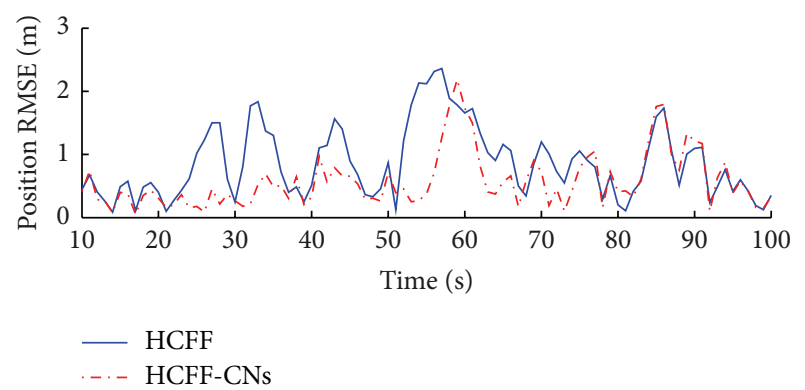

(a)

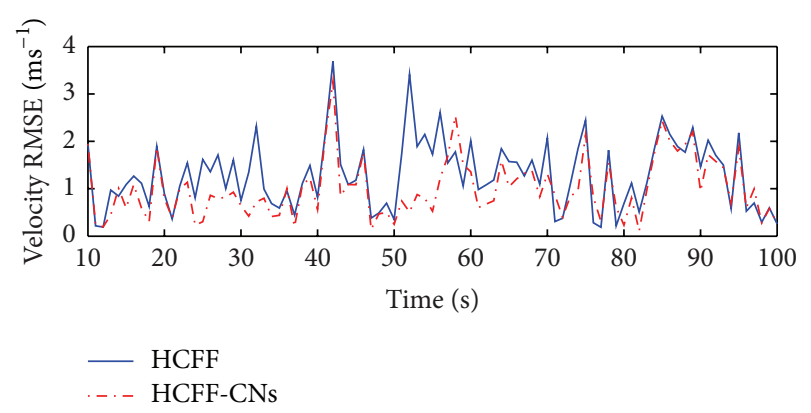

(b)

FIGURE 4: RMSEs in position and velocity for Scenario 2.

TABLE 2: Means of RMSEs in position and velocity for Scenario 2.

\begin{tabular}{lcc}
\hline Algorithms & HCFF & HCFF-CNs \\
\hline RMSE means in position $(\mathrm{m})$ & 1.0351 & 0.7711 \\
RMSE means in velocity $\left(\mathrm{ms}^{-1}\right)$ & 1.3734 & 1.0284 \\
\hline
\end{tabular}

achieve better accuracy and robustness than HCFF when the process noise and measurement noise are cross-correlated.

\section{Competing Interests}

The authors declare that they have no competing interests.

\section{Acknowledgments}

This work was supported by natural science foundation of Guangdong province (No. 2015A030310131), programs for scientific research start-up funds (No. E15031), and project of enhancing school with innovation (Nos. GDOU2015050226 and GDOU2015050243) of Guangdong Ocean University, science and technology project (No. 2016B01137) of Zhanjiang City.

\section{References}

[1] N. A. Carlson, "Federated filter for fault-tolerant integrated navigation systems," in Proceedings of the IEEE Position Location and Navigation Symposium, Orlando, Fla, USA, 1988.

[2] N. A. Carlson, "Federated square root filter for decentralized parallel processors," IEEE Transactions on Aerospace and Electronic Systems, vol. 26, no. 3, pp. 517-525, 1990.
[3] A. Carlson N, "Federated filter for multiplatform track fusion," in Proceedings of the SPIE's International Symposium on Optical Science, Engineering, and Instrumentation, pp. 320-331, International Society for Optics and Photonics, 1999.

[4] Y. K. Xu and X. G. Liang, "Information fusion for radar/infrared compound seeker based on federated filter," International Journal of Digital Content Technology \& Its Applications, vol. 5, pp. 218-229, 2011.

[5] H. Zhao and Z. Li, "Ultra-tight GPS/IMU integration based long-range rocket projectile navigation," Defence Science Journal, vol. 66, no. 1, pp. 64-70, 2016.

[6] X. Gong and J. Zhang, "An innovative transfer alignment method based on federated filter for airborne distributed POS," Measurement, vol. 86, pp. 165-181, 2016.

[7] J. Feng, Z. Wang, and M. Zeng, "Distributed weighted robust Kalman filter fusion for uncertain systems with autocorrelated and cross-correlated noises," Information Fusion, vol. 14, no. 1, pp. 78-86, 2013.

[8] E. Song, Y. Zhu, J. Zhou, and Z. You, "Optimal Kalman filtering fusion with cross-correlated sensor noises," Automatica, vol. 43, no. 8, pp. 1450-1456, 2007.

[9] B. Jia, M. Xin, and Y. Cheng, "High-degree cubature Kalman filter," Automatica, vol. 49, no. 2, pp. 510-518, 2013.

[10] X. R. Li, "Optimal linear estimation fusion-part VII: dynamic systems," in Proceedings of the 6th International Conference on Information Fusion (FUSION '03), vol. 1, pp. 455-462, IEEE Press, Cairns, Australia, July 2003.

[11] Y. Shi, L. Che, Q. Ge, and X. Zhou, "Adaptive high-degree Cubature Kalman filter with unknown noise statistics," Journal of Information \& Computational Science, vol. 11, no. 18, pp. 67036712, 2014.

[12] S. S. Wang, G. Q. Qi, and L. J. Wang, "High degree cubature $\mathrm{H}$-infinity filter for a class of nonlinear discrete-time systems," International Journal of Innovative Computing, Information and Control, vol. 11, no. 2, pp. 627-640, 2015.

[13] Z. Hu, M. Qin, J. Wang, and Y. Liu, "Unscented Kalman filter based on the decoupling of correlated noise," Journal of Computational Information Systems, vol. 9, no. 7, pp. 2941-2948, 2013.

[14] X. X. Wang, Y. Liang, Q. Pan, and F. Yang, "A Gaussian approximation recursive filter for nonlinear systems with correlated noises," Automatica, vol. 48, no. 9, pp. 2290-2297, 2012.

[15] X.-X. Wang, L. Zhao, Q.-X. Xia, W. Cao, and L. Li, "Design of unscented Kalman filter with correlative noises," Control Theory and Applications, vol. 27, no. 10, pp. 1362-1368, 2010.

[16] G. Chang, "Comments on 'A Gaussian approximation recursive filter for nonlinear systems with correlated noises' [Automatica 48 (2012) 2290-2297]," Automatica, vol. 50, no. 2, pp. 655-656, 2014.

[17] Q. Ge, D. Xu, and C. Wen, "Cubature information filters with correlated noises and their applications in decentralized fusion," Signal Processing, vol. 94, no. 1, pp. 434-444, 2014. 


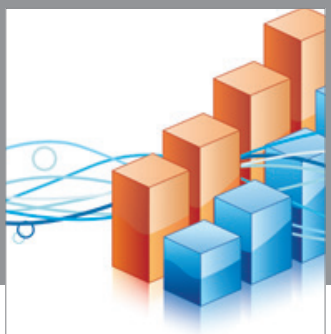

Advances in

Operations Research

vatem alat4

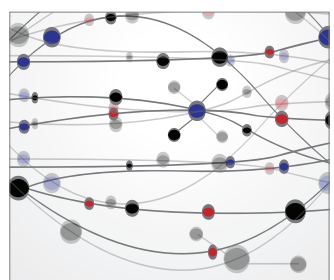

\section{The Scientific} World Journal
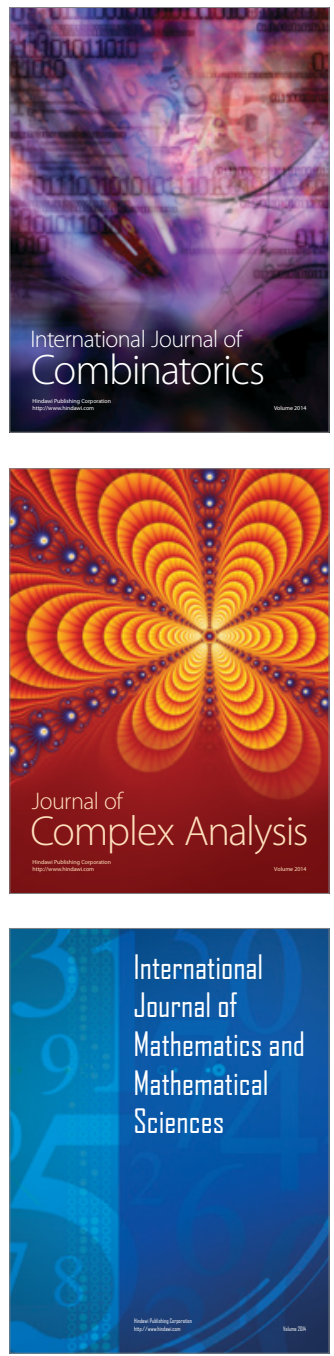
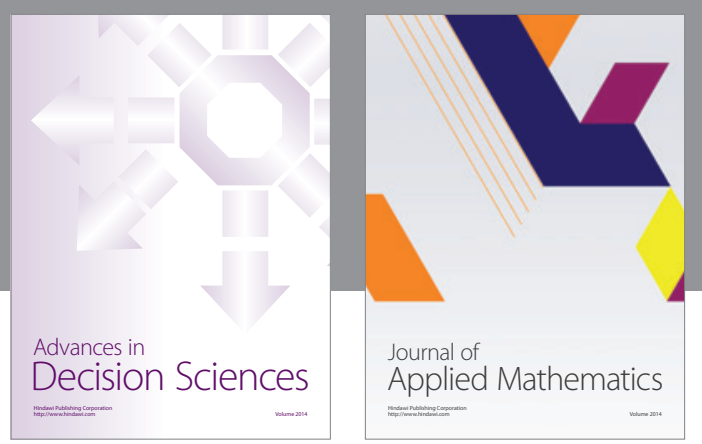

Algebra

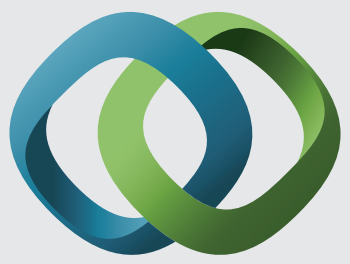

\section{Hindawi}

Submit your manuscripts at

http://www.hindawi.com
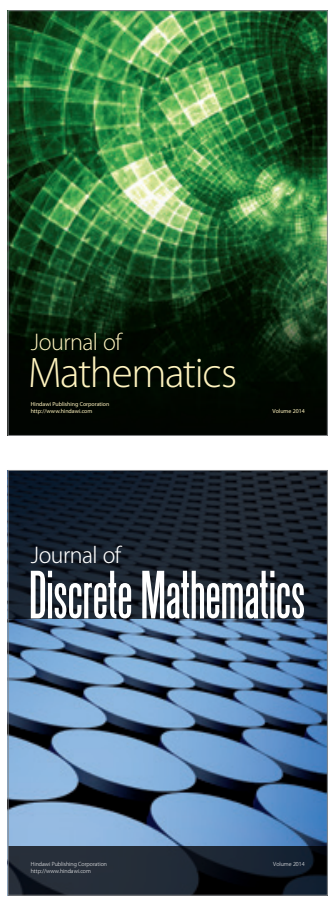

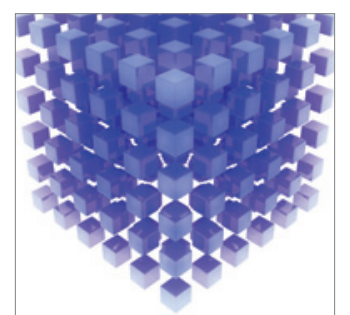

Mathematical Problems in Engineering
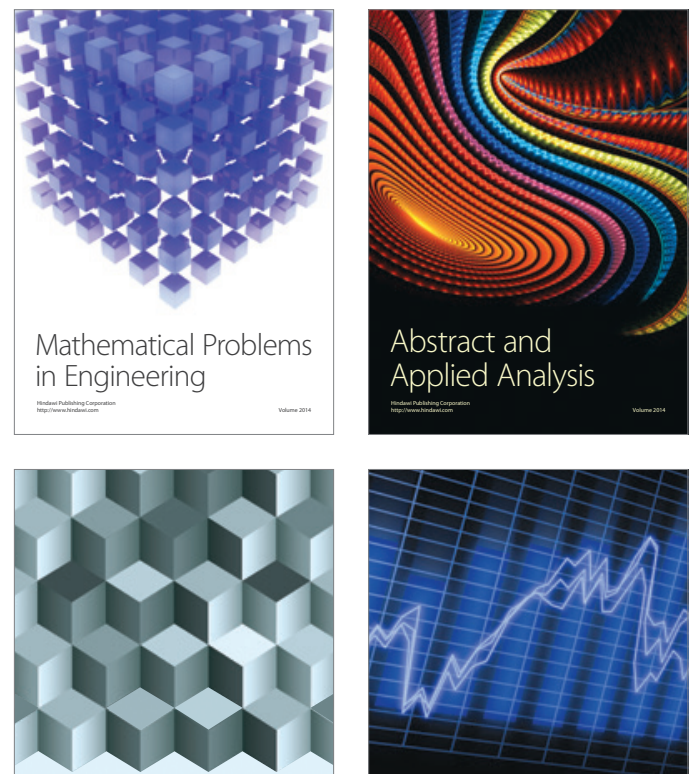

Journal of

Function Spaces

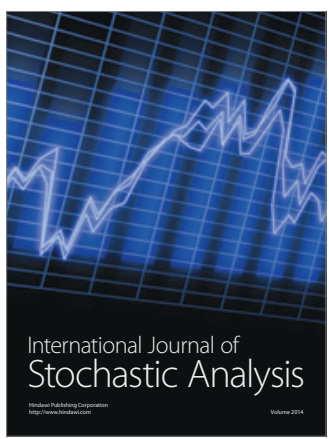

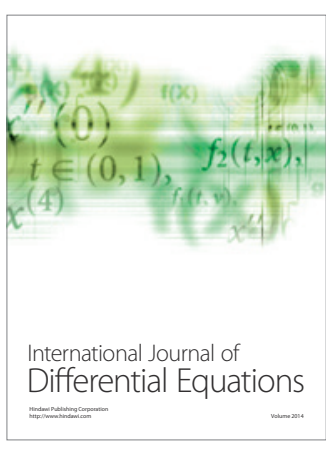
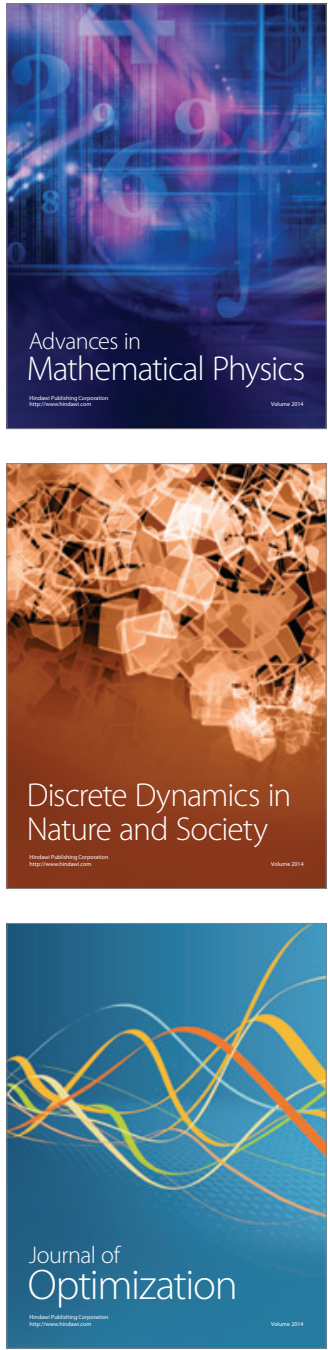